\title{
Sur certains problèmes aux limites discontinues d'ordre supérieur dans la théorie des fonctions analytiques
}

\author{
par W. Pogonzelski (Warszawa)
}

1. Introduction. Dans les travaux [1] et [2] I. N. Vécoua a posé et résolu le problème de la recherche d'une fonction holomorphe, vérifiant une condition limite linéaire concernant cette fonction et quelques unes de ses dérivées. Ce problème a été généralisé par $\mathrm{N}$. P. Vécoua [3] et par B. V. Hvédélidzé [4] au cas où les fonctions figurant dans la condition limite sont discontinues.

Dans ce travail nous allons étudier un problème qui est une extension du problème discontinu de Vécoua au cas d'une condition aux limites non linéaire. Nous introduirons dans ce problème les fonctions discontinues de classes $\mathfrak{W}_{a}^{h}$ et $H_{a}^{h}$ que nous avons définies et étudiées dans les travaux [6], [7], [8].

2. Rappel du problème linéaire de I. N. Vécoua. Nous rappellerons d'abord brièvement l'énoncé et le procédé amenant à la résolution du problème linéaire de Vécoua, en le généralisant pour les fonctions des classes $\mathfrak{H}_{a}^{h}$ et $\boldsymbol{H}_{a}^{h}$.

Soit un domaine borné $S^{+}$limité par une seule courbe fermée $L$ de Jordan. Le problème de Vécoua consiste à déterminer une fonction $\Phi(z)$, holomorphe dans le domaine $S^{+}$et admettant une dérivée $\Phi^{(m)}(z)$ de classe $H_{a}\left(c_{k_{1}}, \ldots, c_{k_{q}}\right)$ relativement aux points de discontinuité $c_{1}, c_{2}, \ldots, c_{p}$ sur $L$, qui vérifie la relation suivante

$$
\text { re } \sum_{j=0}^{m}\left\{a_{j}(t) \Phi^{(j)}(t)+\int_{L} g_{j}(t, \tau) \Phi^{(j)}(\tau) d \sigma\right\}=f(t) \quad(m \geqslant 1)
$$

entre les valeurs limites de la fonction $\Phi(t)=\Phi^{(0)}(t)$ elle-même et de ses dérivées $\Phi^{(j)}(t)$ en tout point $t$ sur $L$ différent des points de discontinuité $c_{1}, \ldots, c_{p}$. Nous supposons vérifiées les conditions suivantes.

A. La fonction réelle $f(t)$, définie sur $L$, appartient à la classe $\mathfrak{H}_{a}^{h}\left(c_{k_{1}}, \ldots, c_{k_{q}}\right)$ relativement aux points de discontinuité $c_{1}, c_{2}, \ldots, c_{p}$, ordonnés suivant le sens positif de la ligne $L$. 
B. Les fonctions complexes $a_{j}(t)$, définies en tout point de la ligne $L$, vérifient une condition de Hölder

$$
\left|a_{j}(t)-a_{f}\left(t_{1}\right)\right|<\text { const }\left|t-t_{1}\right|^{h_{a}} \quad\left(0<h_{a} \leqslant 1\right)
$$

et l'inégalité $a_{m}(t) \neq 0$.

C. Les fonctions complexes $g_{j}(t, \tau)$ s'expriment par la formule

$$
g_{j}(t, \tau)=\frac{g_{j}^{(0)}(t, \tau)}{|t-\tau|^{\nu}} \quad(0 \leqslant \gamma<1)
$$

où les fonctions complexes $g_{f}^{(0)}(t, \tau)$ sont définies pour $t, \tau \in L$ et vérifient une condition de Hölder

$$
\left|g_{j}^{(0)}(t, \tau)-g_{j}^{(0)}\left(t_{1}, \tau_{1}\right)\right|<\operatorname{const}\left[\left|t-t_{1}\right|^{h_{a}}+\left|\tau-\tau_{1}\right|^{h_{a}}\right] .
$$

D. La ligne fermée $L$ a une tangente continue en tout point et l'angle $\Delta\left(t, t_{1}\right)$ entre les tangentes en deux points arbitraires $t$ et $t_{1}$ vérifie l'inégalité

$$
\Delta\left(t, t_{1}\right)<\text { const }\left|t-t_{1}\right|^{h_{L}} \quad\left(0<h_{L} \leqslant 1\right) .
$$

On cherche la solution du problème posé moyennant la formule intégrale de Vécoua

$$
\Phi(z)=\int_{\Sigma}(1-z / \tau)^{m-1} \log (1-z / \tau) \mu(\tau) d \sigma+\int_{\Sigma} \mu(\tau) d \sigma+i C,
$$

où $\mu(\tau)$ est une fonction inconnue réelle de classe $\mathfrak{S}_{a}\left(c_{k_{1}}, \ldots, c_{k_{q}}\right), C$ une constante inconnue réelle, $\sigma$ l'abscisse curviligne du point d'intégration $\tau$ $\operatorname{sur} L$. Dans la formule (6) on prend la branche de la fonction logarithmique qui s'annule au point $z=0$, supposé à l'intérieur du domaine $S^{+}$.

De la formule (6) on obtient les expressions suivantes des dérivées de la fonction $\Phi(z)$ en tout point $z \in \mathbb{S}^{+}$

où l'on a posé

$$
\Phi^{(j)}(z)=\int_{\boldsymbol{L}} N_{j}(z, \tau) \mu(\tau) d \sigma \quad(j=1,2, \ldots, m),
$$

$$
\begin{gathered}
N_{f}(z, \tau)=(-1)^{j} \tau^{-j}(m-1) \ldots(m-j)\left(1-\frac{z}{\tau}\right)^{m-j-1} \times \\
\times\left[\log \left(1-\frac{z}{\tau}\right)+\frac{1}{m-1}+\ldots+\frac{1}{m-j}\right] \quad(j=1,2, \ldots, m-1), \\
N_{m}(z, \tau)=\frac{(-1)^{m}(m-1) !}{\tau^{m-1}(\tau-z)} .
\end{gathered}
$$

En demandant que la fonction (6) et ses dérivées (7) vérifient la condition limite (1) en tout point $t \neq c_{\text {, }}$ sur $L$, en nous appuyant sur les formules de Plemelj et sur la transformation connue de Poincaré de l'intégrale 
singulière itérée (valable aussi pour les fonctions de classe $\mathfrak{h}$ ), nous obtenons l'équation intégrale singulière suivante avec la fonction inconnue $\mu(t)$ :

$$
A(t) \mu(t)+\int_{L} N(t, \tau) \mu(\tau) d \sigma=f(t)-C w(t) .
$$

Les fonctions réelles $A(t), \dot{N}(t, \tau), w(t)$ sont définies par les formules suivantes, empruntées à la belle monographie de Mouskhelichvili ([5], p. 213):

$$
\begin{gathered}
A(t)=\frac{1}{2}(-1)^{m}(m-1) ! \pi i\left[t^{1-m} \bar{t} a_{m}(t)-\bar{t}^{1-m} t^{\prime} \overline{a_{m}(t)}\right], \\
w(t)=\mathrm{re}\left[i a_{0}(t)+i \int_{\bar{L}} g_{0}(t, \tau) d \sigma\right], \\
N(t, \tau)=\sum_{j=0}^{m} \operatorname{re}\left\{a_{j}(t) N_{j}(t, \tau)+\int_{L} g_{j}\left(t, \tau_{1}\right) N_{j}\left(\tau_{1}, \tau\right) d \sigma_{1}\right\} .
\end{gathered}
$$

Mettant en évidence la partie singulière, nous pouvons écrire l'équation (9) sous la forme équivalente (voir [5], p. 215)

$$
A(t) \mu(t)+\frac{1}{\pi i} B(t) \int_{\Sigma} \frac{\mu(\tau) d \tau}{\tau-t}+\int_{L} \frac{k(t, \tau) \mu(\tau)}{|t-\tau|^{\beta}} d \sigma=f(t)-C w(t)=\tilde{f}(t),
$$

où l'on a posé

$$
B(t)=\frac{1}{2}(-1)^{m}(m-1) ! \pi i\left[t^{1-m} \bar{t}^{\prime} a_{m}(t)+\bar{t}^{1-m} t^{\prime} \overline{a_{m}(t)}\right] .
$$

Les fonctions $A(t)$ et $B(t)$, définies sur $L$, vérifient une condition de Hölder d'exposant

$$
h_{a}^{\prime}=\min \left(h_{a}, h_{L}\right) .
$$

La fonction complexe $k(t, \tau)$, définie pour $t, \tau \in L$, vérifie une condition de Hölder

$$
\left|k(t, \tau)-k\left(t_{1}, \tau_{1}\right)\right|<\operatorname{const}\left[\left|t-t_{1}\right|^{\beta}+\left|\tau-\tau_{1}\right|^{\chi}\right] .
$$

Les exposants positifs $\beta$ et $\chi$, inférieurs à l'unité, sont déterminés par les formules

$$
\beta=1-\theta h_{L}, \quad \chi=\min \left[h_{a}^{\prime}, \theta(1-\gamma),(1-\theta) h_{L}\right],
$$

où $\theta$ est une constante positive arbitraire, inférieure à l'unité. En vertu des formules $\left(10^{\prime}\right)$ et (11), la fonction réelle connue $\tilde{f}(t)$ appartient à la classe $\mathfrak{H}_{a}^{h_{1}}\left(c_{k_{1}}, \ldots, c_{\boldsymbol{k}_{q}}\right)$, où

$$
h_{1}=\min \left[h, h_{a}, \theta(1-\gamma)\right] \text {. }
$$

Pour ramener l'équation (1) à une équation de Fredholm, on sait qu'il faut d'abord étudier l'équation caractéristique

$$
A(t) \mu(t)+\frac{1}{\pi i} B(t) \int_{L} \frac{\mu(\tau) d \tau}{\tau-t}=F(t)
$$


où $F(t)$ est une fonction complexe, donnée sur $L$, appartenant à la classe $\mathfrak{S}_{a}^{r}\left(c_{k_{1}}, \ldots, c_{k_{q}}\right)$. On trouve la solution de l'équation (17) d'une façon connue, en résolvant un problème de Hilbert avec la condition limite

$$
\Psi^{+}(t)=\frac{A-B}{A+B} \Psi^{-}(t)+\frac{F(t)}{A+B}
$$

en tout point $t \neq c$ de la ligne $L$, où $A^{2}-B^{2} \neq 0$ à cause de l'hypothèse $a_{m}(t) \neq 0$. On obtient ainsi la solution générale de l'équation (17) sous la forme

$$
\begin{aligned}
\mu(t)=\frac{X^{+}(t)-X^{-}(t)}{2 \pi i} \int_{L} \frac{F(\tau) d \tau}{[A(\tau)+B(\tau)] X^{+}(\tau)(\tau-t)}+ \\
+\frac{A(t)}{A^{2}(t)-B^{2}(t)} F(t)+\left[X^{+}(t)-X^{-}(t)\right] P_{\varkappa-1}(t),
\end{aligned}
$$

l'indice du problème $x$ étant supposé non négatif:

$$
\varkappa=\frac{1}{2 \pi}\left[\frac{A-B}{A+B}\right]=2 m+\frac{1}{\pi}\left[\arg a_{m}(t)\right]_{L} \geqslant 0 ;
$$

$X^{ \pm}(t)$ désignent les fonctions limites de la solution canonique $X(z)$ du problème (18), partout différentes de zéro, $P_{x-1}$ est un polynôme arbitraire de degré $x$, si $x>0$, et $P=0$, si $x=0$. En vertu des propriétés des fonctions de classe $\mathfrak{S}$, la fonction (19) appartient à la classe $\mathfrak{S}_{a}^{r_{1}}\left(c_{k_{1}}, \ldots, c_{k_{q}}\right)$, où

$$
r_{1}=\min \left(r, h_{a}^{\prime}\right)
$$

En nous appuyant sur la solution (19), nous pouvons affirmer, dans le cas où $x \geqslant 0$, que l'équation intégrale singulière (13) ou (15) est équivalente à l'équation de Fredholm

où l'on a posé

$$
\mu(t)=\int_{i} M(t, \tau) \mu(\tau) d \sigma+\widetilde{F}(t)
$$

$$
\begin{array}{r}
\text { (21) } \quad M(t, \tau)=-\frac{X^{+}(t)-X^{-}(t)}{2 \pi i} \int_{\Sigma} \frac{k(\zeta, \tau) d \tau}{[A(\zeta)+B(\zeta)] X^{+}(\zeta)(\zeta-t)|\zeta-\tau|^{\beta}}- \\
-\frac{A(t)}{A^{2}(t)-\bar{B}^{2}(t)} \cdot \frac{k(t, \tau)}{|t-\tau|^{\beta}}, \\
\text { (22) } \quad F(t)=\frac{X^{+}(t)-X^{-}(t)}{2 \pi i} \int_{L} \frac{\tilde{f}(\tau) d \tau}{[A(\tau)+B(\tau)] X^{+}(\tau)(\tau-t)}+ \\
+\frac{A(t) \tilde{f}(t)}{A^{2}(t)-B^{2}(t)}+\left[X^{+}(t)-X^{-}(t)\right] P_{x-1}(t) .
\end{array}
$$


Le noyau de l'équation intégrale (20) est comparable à la fonction $O\left(|t-\tau|^{-\beta}\right)$, il admet donc une faible singularité. En outre, ce noyau vérifie une condition de Hölder généralisée de la forme

$$
\left|M(t, \tau)-M\left(t_{1}, \tau\right)\right|<\frac{\text { const }\left|t-t_{1}\right|^{x}}{|t-\tau|^{\beta+\chi}}
$$

pour $t, t_{1} \neq \tau,|t-\tau|<\left|t_{1}-\tau\right|$. D'après les propriétés des fonctions de classe $\mathfrak{S}$, la fonction (22) appartient a la classe $\mathfrak{S}_{a}^{h_{1}}\left(c_{k_{1}}, \ldots, c_{k_{q}}\right)$. A l'équation (20) on peut appliquer la théorie classique de Fredholm. Notamment, si nous supposons que l'équation homogène

$$
\mu(t)=\int_{L} M(t, \tau) \mu(\tau) d \sigma
$$

ne possède pas de solutions non nulles, alors l'équation (20) admet une solution unique, donnée par la formule $\left(t \neq c_{v}\right)$

$$
\mu^{*}(t)=\int_{\boldsymbol{L}} \mathfrak{M}(t, \tau) \widetilde{F}(\tau) d \tau+\widetilde{F}(t)
$$

où $\mathfrak{M}$ désigne la somme de quelques noyaux itérés et du noyau résolvant. Cette solution appartient à la classe $\mathfrak{S}_{a}^{x}\left(c_{k_{1}}, \ldots, c_{k_{q}}\right)$, en vertu de l'inégalité (23) et des propriétés des fonctions de classe $\mathfrak{H}$.

Il en résulte que la fonction $\mu^{*}(t)$ obtenue est une solution des équations primitives (9) et (11). Remarquant ensuite que les fonctions $A(t)$, $N(t, \tau), f(t)-C w(t)$ dans l'équation (9) sont réelles, nous conclnons que la partie réelle

$$
\mu^{\mathrm{re}}(t)=\operatorname{re}\left[\mu^{*}(t)\right] \in \mathfrak{S}_{a}^{\chi}\left(c_{k_{1}}, \ldots, c_{k_{q}}\right)
$$

de la solution (25) de l'équation (20) est aussi une solution de cette équation. Les coefficients du polynôme $P_{x^{-1}}(t)$ étant arbitraires, cette solution s'exprime par une combinaison linéaire (voir [5], p. 217)

$$
\mu^{\mathrm{re}}(t)=\mu_{0}(t)-C \tilde{\mu_{0}}(t)+\sum_{\nu=1}^{n} C_{\nu} \mu_{\nu}(t)
$$

de certaines fonctions réelles à coefficients réels arbitraires. En substituant la fonction (27) dans l'expression (6), nous obtenons, dans le cas où $x \geqslant 0$, la solution cherchée $\Phi(z)$ du problème linéaire, dont la dérivée $\Phi^{(m)}(z)$ appartient à la classe $H_{a}^{x}\left(c_{k_{1}}, \ldots, c_{k_{q}}\right)$.

3. Énoncé et résolution du problème non linéaire. Lo problème aux limites discontinues, non linéaires, que nous proposons de résoudre, consiste à déterminer une fonction $\Phi(z)$, holomorphe dans le domaine $S^{+}$et admettant une dérivée $\Phi^{(m)}(z)$ de certaine classe $H_{a}^{o}$ rela- 
tivement aux points de discontinuité $c_{1}, c_{2}, \ldots, c_{p}$ sur $L$, dont les fonctions limites vérifient la relation non linéaire

$$
\begin{aligned}
\operatorname{re} \sum_{j=0}^{m}\left\{a_{f}(t) \Phi^{(f)}(t)+\int_{L} g_{f}(t, \tau) \Phi^{(j)}(\tau) d \sigma\right\} & \\
& =F\left[t, \Phi(t), \Phi^{\prime}(t), \ldots, \Phi^{(m)}(t)\right]
\end{aligned}
$$

en tout point $t \in L-\sum_{i=1}^{p} c_{\nu}$ (les constantes $a$ et $\delta$ seront fixées plus loin).

On admet pour les fonctions $a_{j}(t), g_{j}(t, \tau)$ et pour la ligne fermée $L$ les mêmes hypothèses que précédemment. On suppose que la fonction réelle $F\left(t, u_{0}, u_{1}, \ldots, u_{m}\right)$ soit définie dans la région

$$
t \in L-\sum_{r=1}^{p} c_{\eta}, \quad u_{p} \in \Pi \quad(\nu=0,1, \ldots, m)
$$

où $\Pi$ désigne le plan entier de la variable complexe. On admet ensuite que la fonction $F$ vérifie l'inégalité

$$
\left|F^{\prime}\left(t, u_{0}, u_{1}, \ldots, u_{m}\right)\right|<k_{F} \sum_{p=0}^{m}\left|u_{v}\right|+\frac{M_{F}}{\prod_{p=1}^{p}\left|t-c_{\nu}\right|^{n}}
$$

et la condition de Hölder généralisée suivante

$$
\begin{aligned}
& \left|F\left(t, u_{0}, u_{1}, \ldots, u_{m}\right)-F\left(t^{\prime}, u_{0}^{\prime}, u_{1}^{\prime}, \ldots, u_{m}^{\prime}\right)\right| \\
< & k_{F}\left[\frac{\left|t-t^{\prime}\right|^{h_{F}}}{\left|t-c_{j}\right|^{\alpha+h_{F}}\left|t^{\prime}-c_{j+1}\right|^{a+h_{F}}}+\sum_{i=0}^{m}\left|u_{\nabla}-u_{*}^{\prime}\right|\right] \quad\left(t \in \widehat{c_{j} c_{j+1}}, t^{\prime} \in \widehat{t c_{j+1}}\right) .
\end{aligned}
$$

La fonction $F$ est donc de classe $\mathfrak{S}_{a}^{h_{F}}$ par rapport à la variable $t$.

De même que précédemment, nous allons chercher la solution du problème sous forme d'une intégrale de Vécoua

$$
\Phi(z)=\int_{L} \mu(\tau)(1-z / \tau)^{m-1} \log (1-z / \tau) d \sigma+\int_{L} \mu(\tau) d \sigma+i C
$$

$\mu$ étant une fonction inconnue réelle de classe $\mathfrak{S}_{a}, C$ une constante réelle. inconnue.

En demandant que la fonction (32) vérifie la condition. limite (28), on arrive, en suivant les considérations développées dans l'article précédent, à l'équation intégrale singulière suivante

$$
\begin{aligned}
& A(t) \mu(t)+\int_{L} N(t, \tau) \mu(\tau) d \sigma=-C w(t)+ \\
+ & F\left[t, Z_{0}(t, \mu), Z_{1}(t, \mu), \ldots, Z_{m-1}(t, \mu) ; b(t) \mu(t)+\frac{1}{\pi i} \int_{L} \frac{b(\tau) \mu(\tau) d \tau}{\tau-t}\right]
\end{aligned}
$$


où les fonctions réelles connues $A(t), N(t, \tau), v(t)$, sont données par les formules $(\mathbf{1 0}),\left(10^{\prime}\right),\left(10^{\prime \prime}\right)$, la fonction connue $b(t)$ est donnée par la formule

$$
b(t)=(-1)^{m}(m-1) ! \pi i t^{1-m} \bar{t}^{\prime}
$$

et enfin les fonctionnelles $Z_{0}(t, \mu), \ldots, Z_{m-1}(t, \mu)$ sont liées à la fonction réelle inconnue $\mu(t)$ par les formules intégrales

$$
\begin{aligned}
Z_{j}(t, \mu)=(-1)^{j}(m-1) & \ldots(m-v) \int_{L}\left(1-\frac{t}{\tau}\right)^{m-j-1} \times \\
\times & {\left[\log \left(1-\frac{t}{\tau}\right)+\frac{1}{m-1}+\ldots+\frac{1}{m-j}\right] \mu(\tau) d \sigma }
\end{aligned}
$$

à faibles singularités $(j=0,1, \ldots, m-1)$. Le terme contenant l'intégrale à singularité forte est écrit explicitement dans l'équation (33). D'après la théorie du problème linéaire, toute fonction complexe $\varphi(t)$ de classe $\mathfrak{S}_{a}$ qui vérifie l'équation

$$
\begin{aligned}
& \boldsymbol{A}(t) \varphi(t)+\int_{L} N(t, \tau) \varphi(\tau) d \sigma=-C w(t)+ \\
& \quad+F\left[t, Z_{0}(t, \mu), \ldots, Z_{m-1}(t, \mu) ; b(t) \mu(t)+\frac{1}{\pi i} \int_{L} \frac{b(\tau) \mu(\tau) d \tau}{\tau-t}\right]
\end{aligned}
$$

étant une forme généralisée de l'équation (33), où $\mu(t)=\mathrm{re}[\varphi(t)]$, vérifie aussi l'équation intégrale suivante

$$
\begin{array}{r}
\varphi(t)=\int_{L} M(t, \tau) \varphi(\tau) d \sigma_{\tau}+\int_{L} G(t, \tau) F\left[\tau, Z_{0}(\tau, \mu),\right. \\
\left.\ldots, Z_{m-1}(\tau, \mu) ; b(\tau) \mu(\tau)+\frac{1}{\pi i} \int_{L} \frac{b(\zeta) \mu(\zeta) d \zeta}{\zeta-\tau}\right] \frac{d \tau}{\tau-t}+ \\
+\frac{A(t)}{A^{2}(t)-B^{2}(t)} F\left[t, Z_{0}(t, \mu), \ldots, Z_{m-1}(t, \mu) ; b(t) \mu(t)+\frac{1}{\pi i} \int_{\tilde{L}} \frac{b(\tau) \mu(\tau) d \tau}{\tau-t}\right]+ \\
+C \tilde{w}(t)+\left[X^{+}(t)-X^{-}(t)\right] P_{*-1}(t),
\end{array}
$$

où $X^{+}(t)$ et $X^{-}(t)$ sont les fonctions limites de la solution canonique $X \dot{(z)}$ d'indice $x \geqslant 0$. Le noyau $M(t, \tau)$, à singularité faible, est défini par la formule (21). $G$ et $\tilde{w}$ sont des fonctions connues, définies par les formules

$$
\begin{gathered}
G(t, \tau)=\frac{X^{+}(t)-X^{-}(t)}{2 \pi i X^{+}(\tau)[A(\tau)+B(\tau)]}, \\
\tilde{w}(t)=\frac{X^{+}(t)-X^{-}(t)}{2 \pi i} \int_{L} \frac{w(\tau) d \tau}{[A(\tau)+B(\tau)] X^{+}(\tau)} \overline{(\tau-t)}-\frac{A(t) w(t)}{A^{2}(t)-B^{2}(t)}
\end{gathered}
$$


$w(t)$ étant donnée par la formule $\left(10^{\prime}\right), P_{x-1}(t)$ est un certain polynôme de degré $x$ au plus, ou $P=0$ si $x=0$.

Considérons maintenant dans (37) la constante $C$ et les coefficients du polynôme $P_{x-1}(t)=C_{1}+C_{2} t+\ldots+C_{x} t^{x-1}$ comme des constantes arbitraires et cherchons la solution $\varphi(t)$ de l'équation intégrale (37). Observons que dans cette équation les singularités fortes interviennent doublement dans le second terme de la somme (37).

L'équation (37) étant irrésoluble par les méthodes de l'Analyse classique, nous allons en chercher la solution par l'application du théorème topologique connu de J. Schauder [9]:

Si, dans un espace de Banach une transformation continue fait correspondre d un ensemble de points, convexe et fermé, son sous-ensemble compact, alors il existe dans l'ensemble donné au moins un point invariant de la transformation.

Soit donc $A$ l'espace fonctionnel composé de toutes les fonctions continues complexes $[\varphi(t)]$ définies dans l'ensemble $L^{\prime}=L-\sum_{p=1}^{p} c_{\nu}$ et vérifiant l'inégalité

$$
\sup _{t \in L^{\prime}}\left[\prod_{\nu=1}^{p}\left|t-c_{v}\right|^{a+0} \cdot|\varphi(t)|\right]<\infty,
$$

où $a$ est la constante positive qui figure dans l'hypothèse (30), la constante positive $\delta$ verifie les inégalités

$$
\delta \leqslant \min \left(h_{F}, \chi\right), \quad a+\delta<1
$$

(voir (15)). On définit ensuite la norme des éléments $[\varphi(t)]$ par la formule

$$
\|\varphi(t)\|=\sup _{t \in L^{\prime}}\left[\prod_{r=1}^{p}\left|t-c_{\boldsymbol{p}}\right|^{a+\delta}|\mu(t)|\right]
$$

et la distance des éléments $\varphi_{1}(t)$ et $\varphi_{2}(t)$ par la formule

$$
\delta\left(\varphi_{1}, \varphi_{2}\right)=\left\|\varphi_{1}(t)-\varphi_{2}(t)\right\| .
$$

On voit que l'espace $\Lambda$ est linéaire, métrique, normé et complet, il est donc un espace de Banach. Considérons maintenant dans l'espace $\Lambda$ l'ensemble $E$ de tous les points $[\varphi(t)]$ vérifiant les inégalités

$$
\begin{gathered}
\prod_{p=1}^{p}\left|t-c_{\nu}\right|^{a} \varphi(t) \mid \leqslant \varrho_{\varphi}, \\
\left|t-c_{f}\right|^{a+\delta} \cdot\left|t_{1}-c_{j+1}\right|^{a+\delta}\left|\varphi(t)-\varphi\left(t_{1}\right)\right| \leqslant x_{\varphi}\left|t-t_{2}\right|^{\delta}
\end{gathered}
$$

$\left(\varphi(t)\right.$ sont donc de classe $\left.\mathfrak{H}_{a}^{\delta}\right)$ les points $t$ et $t_{1}$ étant situés sur le même arc arbitraire $\widehat{c_{j} c_{j+1}}$ entre deux points de discontinuité consécutifs $\left(t_{1} \in \widehat{t_{c_{j+1}}}\right)$; 
$\varrho_{\bar{T}}$ et $x_{\varphi}$ sont des constantes positives, fixées arbitrairement. On montre sans peine que l'ensemble $E$ est fermé et convexe.

En tenant compte de la forme de l'équation intégrale (37), transformons l'ensemble $E$ à l'aide de la relation suivante (où la constante réelle $C$ et les coefficients du polynôme $P_{x-1}(t)$ sont fixés arbitrairement):

$$
\begin{aligned}
& \text { (42) } \quad \psi(t)=\int_{L} M(t, \tau) \psi(\tau) d \sigma+ \\
& \left.+\int_{L} G(t, \tau) F\left[\tau, Z_{0} \tau, \mu\right), \ldots, Z_{m-1}(\tau, \mu) ; b(\tau) \mu(\tau)+\frac{1}{\pi i} \int_{L} \frac{b(\zeta) \mu(\zeta) d \zeta}{\zeta-\tau}\right] \frac{d \zeta}{\tau-t}+ \\
& +\frac{A(t)}{A^{2}(t)-B^{2}(t)} F\left[t, Z_{0}(t, \mu), \ldots, Z_{m-1}(t, \mu) ; b(t) \mu(t)+\frac{1}{\pi i} \int_{L} \frac{b(\tau) \mu(\tau) d \tau}{\tau-t}\right]+ \\
& +C \tilde{w}(t)+\left[X^{+}(t)-X^{-}(t)\right] P_{x-1}(t)
\end{aligned}
$$

où $\mu(t)=\mathrm{re}[\varphi(t)]$ et cherchons une condition pour que cette relation fasse correspondre à tout point $[\varphi(t)]$ de l'ensemble $E$ un point $[\psi(t)]$ du même ensemble. Nous admettons, de même que précédemment, l'hypothèse que l'équation intégrale homogène

$$
\psi(t)=\int_{L} M(t, \tau) \psi(\tau) d \tau
$$

n'a qu'une solution nulle $\psi=0$. Cherchons d'abord les limitations pour le résultat de l'opération suivante sur les fonctions $\mu(t) \in \mathfrak{W}_{a}^{\delta}$, vérifiant aussi les inégalités (41), .

$$
\Xi(t, \mu)=F\left[t, Z_{0}(t, \mu), \ldots, Z_{m-1}(t, \mu) ; b(t) \mu(t)+\frac{1}{\pi i} \int_{L}^{b} \frac{b(\tau) \mu(\tau) d \tau}{\tau-t}\right]
$$

D'après le théorème principal sur les fonctions de classe $\mathfrak{Y}_{a}^{o}$ (voir [6]) et en vertu des inégalités (41), la fonction

$$
\Theta(t)=b(t) \mu(t)+\frac{1}{\pi i} \int_{L} \frac{b(\tau) \mu(\tau) d \tau}{\tau-t}
$$

appartient aussi à la classe $\mathfrak{S}_{a}^{\delta}$ et vérifie les inégalités

$$
\begin{gathered}
|\Theta(t)|<\frac{k_{1} \varrho_{\varphi}+k_{2} \varkappa_{\varphi}}{p}, \\
\prod_{\nu=1}^{p}\left|t-c_{\nu}\right|^{a} \\
\left|\Theta(t)-\Theta\left(t_{1}\right)\right|<\frac{\left(k_{1}^{\prime} \varrho_{\varphi}+k_{2}^{\prime} \varkappa_{\varphi}\right)\left|t-t_{1}\right|^{\delta}}{\left[\left|t-c_{j}\right|\left|t_{1}-c_{j+1}\right|\right]^{a+\delta}}
\end{gathered}
$$


sur tout arc $\widehat{c}_{j} \widehat{c}_{j+1}$, où $k_{1}, k_{2}, k_{1}^{\prime}, k_{2}^{\prime}$ sont des constantes positives indépendantes de la fonction $\mu$. Ensuite, on montre sans peine que les fonctions (46) vérifient les inégalités

$$
\left|Z_{f}(t, \mu)\right|<k_{3} \varrho_{\varphi}, \quad\left|Z_{j}(t, \mu)-Z_{j}\left(t_{1}, \mu\right)\right|<k_{3}^{\prime} \varrho_{\varphi}\left|t-t_{1}\right|^{h_{0}},
$$

$h_{0}$ étant un nombre positif arbitraire, inférieur aux deux nombres $a$ et $1-\alpha, k_{3}$ et $k_{a}^{\prime}$ sont des constantes positives, indépendantes de la fonction $\mu$ et dépendant du choix de $h_{0}$. En tenant compte des propriétés (31), (46), (47), nous concluons que la fonction (44) vérifie les inégalités

$$
\begin{gathered}
|\Xi(t, \mu)|<\frac{\left(\widetilde{C}_{1} \varrho_{\varphi}+\widetilde{C}_{2} \varkappa_{\varphi}\right) k_{F}+\widetilde{C}_{3} M_{F}}{\prod_{p=1}^{p}\left|t-c_{p}\right|^{a}} \\
\left|\Xi(t, \mu)-\Xi\left(t_{1}, \mu\right)\right|<\frac{k_{F}\left(\widetilde{C}_{1}^{\prime} \varrho_{\varphi}+\widetilde{C}_{2}^{\prime} \varkappa_{\varphi}\right)\left|t-t_{1}\right|^{o}}{\left[\left|t-c_{j}\right|\left|t_{1}-c_{j+1}\right|\right]^{a+\delta}}
\end{gathered}
$$

$\widetilde{C_{1}}, \widetilde{C_{2}}, \widetilde{C_{3}}, \widetilde{C_{1}^{\prime}}, \widetilde{C_{2}^{\prime}}$ étant des constantes positives. $\Pi$ en résulte, d'après la propriété principale de la classe $\mathfrak{H}$, que la seconde des intégrales $d u$ second membre de l'équation (42) vérifie aussi inégalités de la forme (48). Nous en déduisons, en vertu des considérations sur le problème linéaire, qu'à tout point $[\varphi(t)]$ de l'ensemble $E$ correspond une solution $\psi(t)$ de l'équation (42) de classe $\mathfrak{H}_{a}^{\mathrm{d}}$, notamment vérifiant les inégalités

$$
\begin{gathered}
|\psi(t)|<\frac{\left(C_{1}^{\prime} \varrho_{\varphi}+C_{2}^{\prime} \varkappa_{\varphi}\right) k_{F}+C_{3}^{\prime} M_{F}+C_{4}^{\prime} K_{p}}{\prod_{p=1}^{p}\left|t-c_{v}\right|^{a}}, \\
\left|\psi(t)-\psi\left(t_{1}\right)\right|<\frac{\left(C_{1}^{\prime \prime} \varrho_{\varphi}+C_{2}^{\prime \prime} \varkappa_{\varphi}\right) k_{F}+C_{4}^{\prime \prime} K_{P}}{\left[\left|t-c_{j}\right|\left|t_{1}-c_{j+1}\right|\right]^{a+\delta}}\left|t-t_{1}\right|^{0},
\end{gathered}
$$

$C_{1}^{\prime}, C_{2}^{\prime}, C_{8}^{\prime}, C_{4}^{\prime}, C_{1}^{\prime \prime}, C_{2}^{\prime \prime}, C_{4}^{\prime \prime}$ étant des constantes positives indépendant de la fonction $F$; la constante positive $K_{P}$ est le plus grand des modules de la constante arbitraire $C$ (voir (37)) et des coefficients arbitraires du polynôme $P_{\kappa-1}(t)$. Les même inégalités (49) évidemment sont vérifiées par la fonction $\mathrm{re}[\psi(t)]$.

En comparant les systèmes d'inégalités (41) et (49), nous concluons que les inégalités

$$
\begin{gathered}
\left(C_{1}^{\prime} \varrho_{\varphi}+C_{2}^{\prime} \varkappa_{\varphi}\right) k_{F}+C_{3}^{\prime} M_{F}+C_{4}^{\prime} K_{P} \leqslant \varrho_{\varphi} \\
\left(C_{1}^{\prime \prime} \varrho_{\varphi}+C_{2}^{\prime \prime} \varkappa_{\varphi}\right) k_{F}+C_{4}^{\prime \prime} K_{P} \leqslant \varkappa_{\varphi}
\end{gathered}
$$

expriment une condition suffisante pour que l'ensemble $E^{\prime}$ des points $[\varphi(t)\rceil$, transformé de l'ensemble $E$ des points $[\varphi(t)]$, fasse partie de cet ensemble. Nous voyons que les inégalités $(\dot{5} 0)$ seront toujours vérifiées, 
si la constante du problème $k_{F}$ est suffisamment petite. Le choix des constantes $\varrho_{\varphi}$ et $\varkappa_{\varphi}$ étant arbitraire, on peut profiter de ce fait et choisir leurs valeurs de façon que l'intervalle de variation permis de la constante $k_{F}$, d'accord avec le système (50), soit le plus grand possible; on obtient $k_{F}<\left(C_{1}^{\prime}+C_{2}^{\prime \prime}\right)^{-1}$. Avant d'appliquer le théorème de Schauder, nous démontrerons encore les deux lemmes suivants.

LEMME 1. La transformation de l'ensemble $E$, définie par la relation (52), est continue dans l'espace $\Lambda$.

Démonstration. Soit une suite arbitraire $\left\{\varphi_{n}(t)\right\}$ de points de l'ensemble $E$ convergente, au sens de la norme (40), vers un point $\varphi(t)$ de cet ensemble. Nous avons donc

$$
\lim _{n \rightarrow \infty}\left\|\varphi_{n}(t)-\varphi(t)\right\|=\lim _{n \rightarrow \infty}\left[\sup _{L} \prod_{n=1}^{p}\left|t-c_{\nu}\right|^{a+\delta}\left|\varphi_{n}(t)-\varphi(t)\right|\right]=0 .
$$

Pour démontrer le théorème, il est nécessaire et suffisant de prouver que la suite $\left\{\psi_{n}(t)\right\}$ de points transformés par la relation (42) converge vers un point $\psi(t)$, qui correspond au point limite $\varphi(t)$ par la relation (42).

Écrivons la relation (42) sous la forme abrégée

$$
\psi(t)=\int_{\boldsymbol{L}} M(t, \tau) \psi(\tau) d \sigma+\Omega(t, \mu),
$$

où l'on a désigné par $\Omega(t, \mu)$ le résultat de l'opération fonctionnelle sur la fonction $\mu=\operatorname{re}(\varphi)$, définie par la somme des termes indiquée dans la formule (42). Nous avons donc aussi

$$
\psi_{n}(t)=\int_{\boldsymbol{L}} M(t, \tau) \psi_{n}(\tau) d \sigma+\Omega\left(t, \mu_{n}\right),
$$

où $\mu_{n}=\mathrm{re}\left(\varphi_{n}\right)$. Etudions d'abord la différence

$$
\boldsymbol{\Xi}(t, \mu)-\boldsymbol{\Xi}\left(t, \mu_{n}\right),
$$

en tenant compte des formules (35), (44) et de la propriété admise (31): Nous aurons alors

$$
\begin{aligned}
\mid \Xi(t, \mu)- & \Xi\left(t, \mu_{n}\right)\left|<k_{F} \sum_{\nu=1}^{m-1}\right| Z_{v}(t, \mu)-Z_{v}(t, \mu) \mid+ \\
+ & |b(t)|\left|\mu(t)-\mu_{n}(t)\right|+\frac{1}{\pi}\left|\int_{L} \frac{b(\tau)\left[\mu(\tau)-\mu_{n}(\tau)\right]}{\tau-t} d \tau\right| \\
& <k_{F}\left\{C^{*} \sup _{L}\left|\mu(t)-\mu_{n}(t)\right|+\frac{1}{\pi}\left|\int_{L} \frac{b(\tau)\left[\mu(\tau)-\mu_{n}(\tau)\right]}{\tau-t} d \tau\right|\right\},
\end{aligned}
$$


où $C^{*}$ est une constante positive, indépendante des fonctions $\mu$ et $\mu_{n}$. Il suffit maintenant d'étudier l'intégrale

$$
J_{n}(t)=\int_{L} \frac{b(\tau)\left[\mu(\tau)-\mu_{n}(\tau)\right]}{\tau-t} d \tau
$$

si $n \rightarrow \infty$, en tenant compte de l'hypothèse (51). Soit donc un arc $l=\widehat{l}^{\prime \prime}$, situé sur la ligne fermée $L$, contenant le point $t$ au milieu de sa longueur, si l'arc $l$ est situé tout entier à l'intérieur d'un arc $\widehat{c}_{p} \widehat{c}_{v+1}$, ou bien contenant le point $t$ à son intérieur de façon que la longueur de l'arc $l^{\prime} t$ soit au plus égale à la longueur de l'arc $\widehat{t l^{\prime \prime}}$ si l'une des extrémités, par exemple $l$, coïncide avec l'une des extrémités $c_{\nu}$ des arcs formant la ligne fermée $L$. Décomposons l'intégrale (56) en somme d'intégrales

$$
J_{n}=J_{n}^{(l)}+J_{n}^{(L-l)}
$$

étendues à l'arc $l$ et à la partie restante $L-l$, et écrivons

$$
J_{n}^{(l)}=\int_{i} \frac{b(\tau)\left\{[\mu(\tau)-\mu(t)]-\left[\mu_{n}(\tau)-\mu_{n}(t)\right\} d \tau\right.}{\tau-t}+\left[\mu(t)-\mu_{n}(t)\right] \int_{l} \frac{d \tau}{\tau-t} .
$$

En appliquant les inégalités (51), nous aurons

$$
\begin{array}{r}
\left|J_{n}^{(l)}\right|<\int_{l^{\prime} t} \frac{2 x_{\varphi}|b(\tau)||t-\tau|^{1-\delta} d \sigma_{\tau}}{\left[\left|\tau-c_{j}\right|\left|t-c_{j+1}\right|\right]^{a+\delta}}+\int_{l^{\prime \prime}} \frac{2 x_{\varphi}|b(\tau)||t-\tau|^{1-\delta} d \sigma_{r}}{\left[\left|t-c_{j}\right|\left|\tau-c_{j+1}\right|\right]^{a+\delta}}+ \\
+\frac{2 \varrho_{\varphi}}{\prod_{,=1}^{p}\left|t-c_{\nu}\right|^{a}}\left|\log \frac{t-l^{\prime}}{l^{\prime \prime}-t}\right| .
\end{array}
$$

I en résulte qu'au nombre positif arbitraire $\varepsilon$ on peut faire correspondre un nombre $\eta(\varepsilon)$ tel que pour tout arc $l=l^{\prime} l^{\prime \prime}$ sur $L$, de longueur $\eta(\varepsilon)$, l'inégalité suivante soit vraie

$$
\sup _{t \in L}\left[\prod_{\nu=1}^{p}\left|t-c_{v}\right|^{a+\delta}\left|J_{n}^{(l)}\right|\right]<\varepsilon .
$$

Remarquons maintenant que la distance $|t-\tau|$ admet nne borne inférieure positive si $\tau \in L-l$, donc la longueur $\eta(\varepsilon)$ de l'arc $l$ étant fixée, nous pouvons ensuite, d'après (51), faire correspondre au nombre $\varepsilon$ un indice $N(\varepsilon)$ tel qu'on ait

$$
\sup _{L}\left[\prod_{p=1}^{p}\left|t-c_{v}\right|^{a+d}\left|J_{n}^{(L-l)}\right|\right]<\varepsilon
$$

si $n>N(\varepsilon)$. En somme, on aura

$$
\lim _{n \rightarrow \infty} \sup _{L}\left[\prod_{n=1}^{p}\left|t-c_{v}\right|^{a+d} \mid J_{n}(t)\right]=0
$$


donc, en vertu de l'inégalité (65), on a aussi

$$
\lim _{n \rightarrow \infty} \sup _{t \in L}\left[\prod_{p=1}^{p}\left|t-c_{\nu}\right|^{a+\delta}\left|\Xi(t, \mu)-\Xi\left(t, \mu_{n}\right)\right|\right]=0,
$$

conformément à l'assertion du lemme 1 . Il reste à étudier la différence des intégrales

$$
I_{n}(t)=\int_{L} G(t, \tau)\left[\Xi(\tau, \mu)-\Xi\left(\tau, \mu_{n}\right)\right] \frac{d \tau}{\tau-t} .
$$

En s'appuyant sur les propriétés (48) et (60), on étudiera l'intégrale (61) de même que précédemment et on conclut que

$$
\lim _{n \rightarrow \infty} \sup _{l \in L}\left[\prod_{\nu=1}^{p}\left|t-c_{\nu}\right|^{a+\delta}\left|I_{n}(t)\right|\right]=0 .
$$

En vertu des résultats (60) et (62), nous arrivons à la propriété suivante

$$
\lim _{n \rightarrow \infty} \sup _{t \in L}\left[\prod_{\nu=1}^{p}\left|t-c_{v}\right|^{a+\delta}\left|\Omega(t, \mu)-\Omega\left(t, \mu_{n}\right)\right|\right]=0 .
$$

En nous appuyant maintenant sur nos considérations antérieures, con-cernant l'équation de Fredholm (20), nous arrivons, d'après la propriété (63), à la conclusion suivante pour les solutions des équations (52) et (53)

$$
\lim _{n \rightarrow \infty}\left\|\psi(t)-\psi_{n}(t)\right\|=\lim _{n \rightarrow \infty}\left[\prod_{p=1}^{p}\left|t-o_{\nu}\right|^{a+\delta}\left|\psi(t)-\psi_{n}(t)\right|\right]=0
$$

ce qui établit notre lemme 1.

LEMME 2. L'ensemble $E^{\prime}$, transformé de l'ensemble $E$ par les relations (42), est compact.

Démonstration. Conformément à la définition de la compacité, il faut et il suffit de démontrer que l'on peut toujours extraire de la suite arbitraire $\left\{\psi_{n}(t)\right\}$ de points de l'ensemble $E^{\prime}$ une suite partielle $\left\{\psi_{k_{n}}(t)\right\}$ convergente au sens de la norme (50). Remarquons donc que les éléments de la suite $\left\{\psi_{n}\right\}$, appartenant à l'ensemble $E^{\prime}$, vérifient l'inégalité (voir (59))

$$
\prod_{p=1}^{p}\left|t-c_{v}\right|^{a}\left|\psi_{n}(t)\right| \leqslant \varrho_{\varphi}
$$

Il en résulte qu'au nombre positif arbitraire $\varepsilon$ on peut faire correspondre un ensemble d'ares $\widehat{c_{v} t^{\prime}}$ et $t^{\prime \prime} c_{v+1}$, situés sur $L$, de longueurs $\eta(\varepsilon)>0$ assez petites pour qu'on ait

$$
\prod_{p=1}^{p}\left|t-c_{p}\right|^{\alpha+\delta}\left|\psi_{n}(t)\right|<\varepsilon / 2
$$


quel que soit $n$, si $t \in L_{0}$, où $L_{0}$ désigne l'ensemble de tous les points des arcs $\widetilde{c_{v} t^{\prime}}$ et $\widehat{t}^{\prime \prime} c_{v+1}\left(\nu=1,2, \ldots, p ; c_{p+1}=c_{1}\right)$. L'ensemble $L_{0}$ étant fixé, observons que les fonctions $\psi_{n}(t)$ vérifient, en vertu de la seconde inégalité (69), pour toute valeur $n$ la même inégalité de Hölder avec un coefficient fixé

$$
\left|\psi_{n}(t)-\psi_{n}\left(t_{1}\right)\right|<\mathrm{const}\left|t-t_{1}\right|^{8}
$$

dans la région fermée $L-L_{0}$. Les fonctions $\psi_{n}(t)$ sont donc bornées dans leur ensemble et équicontinues dans la région $L-L_{0}$, par conséquent, en vertu du théorème connu d'Arzelà, il existe une suite partielle $\psi_{k_{n}}(t)$ uniformément convergente dans $L-L_{0}$ au sens habituel.

Nous pouvons donc faire correspondre au nombre $\varepsilon$ un indice $N(\varepsilon)$ tel qu'on ait

$$
M\left|\psi_{k_{n}}(t)-\psi_{\boldsymbol{k}_{\boldsymbol{g}}}(t)\right|<\varepsilon, \quad \text { si } \quad n, s>N(\varepsilon), \quad t \in L-L_{0}
$$

où

$$
M=\sup _{t \in L}\left[\prod_{y=1}^{p}\left|t-c_{v}\right|^{s+\delta}\right]
$$

En somme, d'après (65) et (66), la condition de Cauchy

$$
\left\|\psi_{k_{n}}(t)-\psi_{k_{s}}(t)\right\|<\varepsilon, \quad \text { si } \quad n, s>N(\varepsilon),
$$

est vérifiée, done la suite $\left\{\psi_{k_{n}}(t)\right\}$ est convergente, l'espace $\Lambda$ étant complet. Par conséquent, le lemme 2 est démontré.

Toutes les conditions du théorème cité de Schauder étant vérifiées, nous en déduirons l'existence d'un au moins point $\varphi^{*}(t)$ dans l'ensemble $E$, invariant relativement à la transformation (42). Cette fonction trouvée $\varphi^{*}(t)$ dans la classe $\mathfrak{S}_{a}^{\delta}$ est donc une solution de l'équation intégrale (37). Par conséquent, la partie réelle

$$
\mu^{*}(t)=\operatorname{re}\left[\varphi^{*}(t)\right]
$$

de cette fonction, qui appartient aussi à la classe $\mathfrak{S}_{a}^{0}$, est une solution de l'équation (33). On l'établit en observant que les fonctions $A(t), N(t, \tau)$, $F, w(t)$ et la constante $C$ sont réelles. En substituant la fonction $\mu^{*}(t) \in \mathfrak{S}_{a}^{d}$ dans la formule (32), on obtient une solution

$$
\Phi^{*}(z)=\int_{L} \mu^{*}(\tau)(1-z / \tau)^{m-1} \log (1-z / \tau) d \sigma+\int_{L} \mu^{*}(\tau) d \sigma+i C
$$

du problème aux limites discontinues (28) non linéaire et d'ordre su-

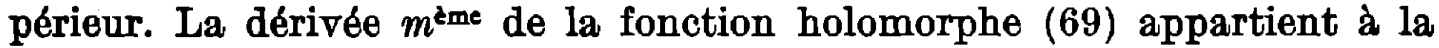
classe $\boldsymbol{H}_{a}^{\boldsymbol{d}}$. Nous pouvons donc énoncer le théorème suivant. 
THÉoRغ̀me. Si les conditions $\mathrm{B}, \mathrm{C}, \mathrm{D}$ de la page 2 sont vérifiées, si l'équation de Fredholm (24) n'admet qu'une solution nulle, si la fonction réelle $F\left(t, u_{0}, \ldots, u_{m}\right)$, définie dans la région (29), vérifie les inégalités (30) et (31), si la constante du problème $k_{F}$ est assez petite pour que les inégalités (50) soient satisfaites, enfin si l'indice $*$ du probleme de Hilbert (18) n'est pas négatif, alors il existe au moins une fonction holomorphe $\Phi^{*}(z)$ dont les valeurs limites et les valeurs limites de ses dérivées, jusqu'à l'ordre $m \geqslant 1$, vérifient la relation non linéaire (28) en tout point $t \epsilon L-\sum_{V} c_{\nu}$. Cette fonction est représentée par la formule $(69)$, où $\mu^{*}(\tau)$ est la partie réelle d'une solution $\varphi^{*}(t)$ de classe $\mathfrak{D}_{a}^{\delta}$ de l'équation intégrale (37).

\section{Travaux cités}

[1] И. Н. Векуа, Об одном новом интегральном представлении аналитических функиий, Сообп. А. Н. Груэ. ССР. т. II, $\mathrm{N}^{0} 6,1941$, p. 477-484, No 8, 1941, p. $701-706$.

[2] - Об одной линейной граничной задаче Римана, Труды Тбилиск. Мат. Инст. T. III, p. 109-139.

[3] Н. П. Векуа, Системы сингулярных интегралных уравнений, Москва 1950.

[4] Б. В. Хведелидзе, Линейные разрывные граничные задаяи теории функиий, сингулярные интегральные уравнения и некоторые их приложсения, Труды Тбилиск. Мат. Инст. T. XXIII, 1956, p. 3-158.

[5] N. I. Muskhelishvili, Singular Integral Équations, Groningen 1953.

[6] W. Pogorzelski, Problèmes aux limites discontinues dans la théorie des fonctions analytiques, Journal of Mathematics and Mechanics, Indiana University, 1960, ou Bull. Acad. Polon. Sci., Sér. des sci. math., astr. et phys. 7 (1950), p. 311.317.

[7] - Sur une propristé principale des fonctions d'une classe 9 de fonctions discontinues, Bull. Acad. Polon. Sci., Sér. des sci. math., astr. et phys. 8 (1960), p. 350-364.

[8] - Propriétés d'une classe de fonctions holomorphes aux fonctions limites discon. tinues, Bull. Acad. Polon. Sci., Sér. des sci. math., astr. et phys. 7 (1959), p. $711-714$ ou Ann. Polon. Math. 9 (1960), p. 189-200.

[9] J. Schauder, Der Fixpunktsatz in Funktionalräumen, Studia Mathematica 2 (1930), p. 171-180.

INSTYTUT MATEMATYCZNY POLSKEEJ AKADEMII NAUK

INSTITUT MATHEMATIQUE DE LACADEMIE POLONAISE DES SCIENCES 